\title{
Aneurysmal bleed in traumatic SAH: are we missing something serious?
}

Suvechha Waiba, Archana Joshi, Karjome Lama, Bidur KC, Bikram Shakya , Amit Thapa

Department of Neurological Surgery, Kathmandu Medical College

Teaching Hospital, Sinamangal, Kathmandu

Correspondence : Dr Amit Thapa, Associate Professor, Department of Neurological Surgery, Kathmandu Medical College Teaching Hospital, Sinamangal, Kathmandu

Email: dramitthapa@yahoo.com

\begin{abstract}
Introduction and Objective: Spontaneous Subarachnoid hemorrhage (SAH) is usually due to aneurysm rupture (85\%) however SAH is far common in traumatic brain injury (TBI) (60\%). We did an audit to see proportion of our patients with TBI with SAH and their outcome

Materials and Methods: We did a retrospective study of patients admitted with Department of neurological surgery since April 2012 till Jan 2016. We included all patients with traumatic SAH and subjected patients with significant cisternal bleed or history of dizziness or headache prior to fall or injury to brain CT angiography. Patient who could not be properly evaluated despite suspicion were excluded. Endpoints studied were outcome, hospital stay and morbidity.
\end{abstract}

Results:112 patients were studied of which 63 were males and mean age was 48.2 years. Total of 5 cases (4.5\%) had intracranial aneurysm. Of these, 3 were PCOM aneurysm and 2 from ACOM. These were successfully clipped and no significant difference in hospital stay or morbidity was seen. Of the other followed up patients, none had recurrent bleed. We discuss our approach and limitations of this study.

Conclusion: $5 \%$ patients with traumatic SAH had aneurysm. A high index of suspicion and early clipping of aneurysm in such cases is associated with good outcome.

Key words: Subarachnoid haemorrhage , Traumatic Brain Injury, outcome 